\title{
Efeitos da cinesioterapia e aconselhamento na qualidade de vida de mulheres incontinentes
}

\author{
Effects of exercise therapy and counseling on the \\ quality of life of incontinent women
}

Carolina Nascimben Matheus Cintia Furlan ${ }^{I I}$

Paloma Felício de Oliveira Lima ${ }^{1}$ Milene Teles Pereira ${ }^{I}$

Paloma Bernardelli Dos Santos ${ }^{1}$ IFaculdades Integradas Einstein de Limeira, Limeira/SP - Brasil IIUniversidade Estadual de Campinas (UNICAMP), Campinas/ SP - Brasil
RESUMo O objetivo deste estudo foi avaliar os efeitos da cinesioterapia e aconselhamento uroginecológico sobre a qualidade de vida de mulheres portadoras de Incontinência Urinária (IU). Participaram deste estudo, 10 mulheres com diagnóstico de IU, divididas em Grupo Cinesioterapia (GC) ( $\mathrm{n}=5 ; 68,2 \pm 6,5$ anos) e Grupo Cinesioterapia + Aconselhamento (GCA) (n=5; 64,6 $\pm 9,8$ anos). Foi aplicado, pré e pós intervenção, o questionário International Consultation on Incontinence Questionnaire - Short Form (ICIQ-SF). O GC realizou 10 sessões em grupo de fortalecimento de tronco, do membro inferior e dos músculos do assoalho pélvico. O GCA recebeu a mesma intervenção, adicionada a aconselhamento (exercícios para casa e diário miccional). O questionário indicou diminuição da frequencia urinária e quantidade das perdas em ambos os grupos, com melhora mais expressiva no GCA. Os dois grupos apresentaram "impacto muito grave" da IU sobre a qualidade de vida antes do tratamento e apenas o grupo aconselhado apresentou melhora, com "impacto moderado" pós tratamento. Podemos inferir que o aconselhamento uroginecológico nos casos de IU pode potencializar os benefícios derivados da cinesioterapia, auxiliando na diminuição da frequencia e quantidade de urina perdida, reduzindo a interferência da IU na vida diária e melhorando a qualidade de vida das mulheres acometidas.

Palavras-Chave: Incontinência urinária; Aconselhamento; EXERCÍCIO TERAPÊUTICO.

Aвstract The aim of this study was to evaluate the effects of kinesiotherapy and urogynecological counseling on the quality of life of women with urinary incontinence (UI). Ten women diagnosed with UI participated in this study. They were divided into Kinesiotherapy Group (KG) $(\mathrm{n}=5 ; 68,2 \pm 6.5$ years $)$ and Kinesiotherapy + Counseling Group (KCG) $(n=5 ; 64,6 \pm 9,8$ years). The volunteers were evaluated pre and post intervention with the International Consultation on Incontinence Questionnaire - Short Form (ICIQ-SF). The KG performed 10 sessions with group activities, including strengthening the trunk and lower limb, and strengthening the pelvic floor muscles. KCG received the same intervention added to counseling (home exercises and voiding diary) in all sessions. The questionnaire indicated decrease in urinary frequency and quantity of losses in both groups, better in GCA. Both groups had "very serious impact" of UI on quality of life before treatment and only the counseled group showed improvement, with "moderate impact" after treatment. We can infer that urogynecological counseling in cases of UI can enhance the benefits derived from kinesiotherapy, helping to decrease the frequency and amount of urine lost, reducing UI interference in daily life and improving the quality of life of affected women.

Keywords: Urinary InCONTINenCE; Counseling; Exercise Therapy. 


\section{INTRODUÇÃO}

Atualmente, a International Continence Society (ICS) define como Incontinência Urinária (IU) a perda involuntária de urina que ocasiona problema social ou higiênico ${ }^{1}$. É um sintoma multifatorial e inúmeras patologias podem provocá-la ${ }^{2}$, sendo as mulheres as mais afetadas ${ }^{3}$.

É considerada uma das mais importantes síndromes geriátricas ${ }^{4}$. Cirurgias, deformidades pélvicas, multiparidade e hipoestrogenismo (característico da menopausa) interferem negativamente na função esfincteriana da bexiga, tornando essa condição mais frequente em mulheres de maior idade ${ }^{5}$.

$\mathrm{O}$ impacto causado pela incontinência afeta negativamente o âmbito sexual, social, doméstico e ocupacional da mulher ${ }^{6}$. Mulheres com IU se sentem envergonhadas, constrangidas para a realização de atividades sociais e esportivas, e com menos desejo para o relacionamento sexual ${ }^{7}$. Há também associação com os sentimentos de solidão e tristeza ${ }^{8}$, além de sintomas depressivos, acompanhados de diminuição da autoestima e aumento da ansiedade?

Considerando todos os sinais e sintomas da IU e sua interferência em diversos aspectos biopsicossociais, a ICS tem recomendado que um questionário de qualidade de vida, como o International Consultation on Incontinence Questionnaire - Short Form (ICIQ-SF), seja incluído em todos os estudos sobre incontinência urinária ${ }^{10}$.

A prevalência da IU é variável em diferentes países devido a diversos fatores, os quais, populações-alvo, características do estudo, ferramentas de avaliação, idade, sexo, disponibilidade de cuidados de saúde, entre outros ${ }^{11}$. Mostafaei et al. ${ }^{12}$, em metanálise, avaliando países de todos os continentes, identificaram que a prevalência total de IU foi de 25,7\%. O Brasil, com cerca de 190 milhões de habitantes, dos quais aproximadamente $56 \%$ são mulheres, apresenta um terço da população feminina acometida pela IU. No entanto, apenas $10,7 \%$ das brasileiras procuram atendimento ginecológico queixando-se de perda urinária ${ }^{13}$.

As principais modalidades terapêuticas conservadoras para o tratamento da IU são: o tratamento farmacológico e a fisioterapia, sendo que ambas buscam amenizar e/ ou curar os sintomas, bem como melhorar a qualidade de vida ${ }^{14}$.

Dentre as diversas técnicas da fisioterapia para reabilitação geniturinária na menopausa, pode-se destacar a cinesioterapia, a qual engloba diversos tipos de exercícios de alongamento e fortalecimento musculares $^{15}$. Considerando a grande influência dos músculos no processo neurofisiológico do controle urinário, estes devem ser fortalecidos para tratamento das disfunções urinárias ${ }^{16,17}$.

Além da intervenção terapêutica por exercícios e treinamento vesical, a conscientização e informação sobre a IU é defendida por vários autores para garantir e prolongar o sucesso da reabilitação ${ }^{18,19}$, entretanto, faltam estudos que mensurem a dimensão da influência do aconselhamento no tratamento dessa afecção. Assim, o objetivo deste estudo foi avaliar os efeitos da cinesioterapia e do aconselhamento uroginecológico sobre a qualidade de vida de mulheres com incontinência urinária. 


\section{MÉTodos}

\subsection{Delineamento do estudo}

A avaliação e a intervenção com a cinesioterapia e aconselhamento uroginecológico foram realizadas nas Faculdades Integradas Einstein de Limeira (FIEL). As voluntárias foram recrutadas por meio de exposição do projeto em mídias sociais e diretamente nas secretarias da saúde de Limeira e Cordeirópolis, as quais possuíam lista de espera de mulheres aguardando atendimento de fisioterapia para IU. Todas as voluntárias assinaram o Termo de Consentimento Livre e Esclarecido (TCLE). Esta pesquisa foi conduzida de acordo com as normas da Resolução 466/12, do Conselho Nacional de Saúde e aprovado pelo Comitê de Ética em Pesquisa das Faculdades Integradas Einstein de Limeira (CEP-FIEL) sob o parecer 3.124.535.

\subsection{Critérios de inclusão e exclusão}

Foram considerados os seguintes critérios de inclusão: 1) apresentar diagnóstico clínico de IU, 2) ser menopausada, 3) apresentar, no mínimo, impacto leve na qualidade de vida em decorrência da IU (pelo menos 1 ponto no ICIQ-SF), 4) ter idade entre 40 e 70 anos.

Foram excluídas as mulheres que tiveram infecção urinária nos últimos seis meses, as que faziam uso de medicamentos que interferiam no trato urinário, as que eram portadoras de patologias neurológicas, as que realizaram tratamento fisioterapêutico para IU nos últimos três meses, as que apresentaram limitações na comunicação ou entendimento relativos à pesquisa e as que se ausentaram da sessão mais do que uma vez.

\subsection{Casuística}

A amostra inicial foi composta por 38 voluntárias, sendo que sete delas não preencheram os critérios de inclusão e 12 apresentaram algum critério de exclusão. Foram divididas, então, 19 mulheres, em dois grupos: Grupo Cinesioterapia $(\mathrm{GC} ; \mathrm{n}=9)$ e Grupo Cinesioterapia + Aconselhamento (GCA; $n=10)$. Durante o período de intervenção, quatro mulheres no GC tiveram duas faltas e foram excluídas do estudo. No GCA, cinco mulheres não finalizaram o estudo, dentre elas, uma sofreu um acidente vascular cerebral, uma abandonou o estudo e três faltaram duas vezes, sendo, portanto, excluídas. Assim, finalizaram este estudo 10 mulheres, cinco no GC e cinco no GCA.

\subsection{Instrumento de avaliação}

Foi realizada uma entrevista de avaliação fisioterapêutica, visando coletar os dados referentes às histórias de saúde das voluntárias, incluindo as características antropométricas e informações sobre o climatério. Foi aplicado também, pré e pós intervenção, o International Consultation on Incontinence Questionnaire - Short Form (ICIQ-SF). Dentre os questionários específicos que avaliam os efeitos da incontinência urinária, o ICIQ-SF foi escolhido por ser simples e autoadministrável, além de avaliar tanto o impacto da IU na qualidade de vida quanto a qualificação da perda urinária das pacientes analisadas ${ }^{10}$.

O ICIQ-SF é composto de quatro questões que avaliam a frequência, a gravidade e o impacto da IU, além de um grupo de oito itens relativos às causas ou às situações de IU vivenciadas pelos respondentes (sendo possível escolher todas as que forem adequadas). 
O escore geral é obtido pela soma dos escores das questões 3, 4 e 5. Quanto maior o escore, maior o impacto sobre a qualidade de vida. $\mathrm{O}$ impacto sobre a qualidade de vida foi dividido da seguinte maneira: nenhum impacto (0 pontos); impacto leve (de 1 a 3 pontos); impacto moderado (de 4 a 6 pontos); impacto grave (de 7 a 9 pontos); e impacto muito grave (10 ou mais pontos $)^{10}$.

\subsection{Intervenção}

A intervenção com cinesioterapia foi realizada em grupo, e 1) composta por fortalecimento de adutores, abdutores, extensores e flexores de quadril, abdominais e extensores de tronco, três séries de 10 a 15 repetições ${ }^{15}$; e 2) pelos exercícios de Kegel - contração dos músculos pélvicos lentamente (contando até cinco) e depois relaxamento (contando até cinco) -, com 10 a 15 repetições, inicialmente em decúbito dorsal e depois em pé16. Foi realizada uma sessão por semana, de 50 minutos, durante 10 semanas, totalizando 10 sessões.

O aconselhamento uroginecológico foi composto por: 1) informações como explicação da anatomia e fisiologia do pavimento pélvico, conscientização dos fatores etiológicos e predisponentes da IU, das formas clínicas, dos tratamentos clínicos e conservadores, além do manejo aos efeitos da IU; 2) estímulo à modificação comportamental (uso de diário miccional, com marcação da ingestão de líquidos e volume urinário); 3) incentivo à continuidade dos exercícios em casa ou no trabalho, incluindo pelo menos oito contrações realizadas três vezes por dia, de modo a evitar o descondicionamento muscular (Kegel) ${ }^{16}$.

\subsection{Análise dos dados}

A análise dos dados foi realizada de maneira descritiva, por meio do Software BioEstat 5.3. Foram apresentadas medidas de tendência central (moda, média e mediana), medidas de variabilidade (desvio padrão e intervalo interquartílico), além de medidas de posição (percentis). Para os dados antropométricos e para a idade, foram utilizadas médias e para os resultados do ICIQ-SF foram utilizadas medianas.

\section{RESUltados}

A média de idade do GC foi de 68,2 \pm 6,5 anos, e do GCA foi de 64,6 \pm 9,8 anos. O Índice de Massa Corporal (IMC) indicou sobrepeso em ambos grupos, com valores médios de $25,86 \pm 1,8 \mathrm{Kg} / \mathrm{m}^{2}$ e $25,17 \pm 4,5$ no GC e no GCA, respectivamente.

Os resultados do ICIQ-SF, do pré e pós tratamento nos dois grupos avaliados, estão apresentados na tabela 1 . Ambos apresentaram diminuição da frequência urinária pós intervenção, porém no GC a moda foi de "diversas vezes ao dia" passando para "uma vez por semana ou menos", e no GCA foi de "diversas vezes ao dia" pré tratamento para frequência bimodal pós que incluiu "uma vez por semana ou menos" e "nunca".

A quantidade de perda urinária também reduziu nos dois grupos, mas foi mais expressiva no GCA que passou de moda "moderada quantidade" de perda urinária para "pequena quantidade".

Nos dois grupos houve redução da interferência da IU na vida diária das participantes, mas no grupo que recebeu aconselhamento essa redução foi de $68,75 \%$ e no grupo sem aconselhamento a redução foi de 37,5\%. 
No que se refere ao ICIQ-SF Escore, os dois grupos apresentaram "impacto muito grave" da IU sobre a qualidade de vida antes do tratamento e apenas o grupo aconselhado apresentou melhora, com "impacto moderado" pós tratamento.

Tanto no GC quanto no GCA houve diminuição do número de situações de perda urinária, principalmente durante a atividade física.

Tabela 1: Frequência e moda das características da IU das voluntárias segundo as questões 3, 4 e 6 do ICIQ-SF, mediana e intervalo interquartílico da questão 5 e do ICIQ-SF Escore, em ambos os grupos.

\begin{tabular}{|c|c|c|c|c|c|}
\hline & \multirow[b]{2}{*}{ Variáveis } & \multicolumn{2}{|c|}{$\mathrm{GC}(\mathrm{n}=5)$} & \multicolumn{2}{|c|}{$\operatorname{GCA}(n=5)$} \\
\hline & & Pré & Pós & Pré & Pós \\
\hline \multirow{6}{*}{$\begin{array}{l}\text { 3. Frequência } \\
\text { de perda } \\
\text { urinária }\end{array}$} & Nunca & 0 & 1 & 0 & $2 *$ \\
\hline & $\begin{array}{l}\text { Uma vez por semana ou } \\
\text { menos }\end{array}$ & 0 & $3 *$ & 0 & $2 *$ \\
\hline & $\begin{array}{l}\text { Duas ou três vezes por } \\
\text { semana }\end{array}$ & 1 & 0 & 1 & 0 \\
\hline & Uma vez ao dia & 0 & 0 & 0 & 0 \\
\hline & Diversas vezes ao dia & $4 *$ & 1 & $4 *$ & 1 \\
\hline & O tempo todo & 0 & 0 & 0 & 0 \\
\hline \multirow{4}{*}{$\begin{array}{l}\text { 4. Quantidade } \\
\text { de perda } \\
\text { urinária }\end{array}$} & Nenhuma & 0 & 1 & 0 & 0 \\
\hline & Pequena quantidade & $3 *$ & $2 *$ & 1 & $4 *$ \\
\hline & Moderada quantidade & 2 & $2 *$ & $3 *$ & 1 \\
\hline & Grande quantidade & 0 & 0 & 1 & 0 \\
\hline \multicolumn{2}{|c|}{$\begin{array}{l}\text { 5. Quanto interfere na vida diária de 0-10 } \\
\text { (mediana e intervalo interquartílico) }\end{array}$} & $8,0(6,0)$ & $5,0(4,0)$ & $8,0(2,2)$ & $2,5(4,7)$ \\
\hline \multicolumn{2}{|c|}{$\begin{array}{l}\text { ICIQ-SF Escore (escore } 3+4+5) \\
\text { (mediana e intervalo interquartílico) } \\
\end{array}$} & $14,0(8,0)$ & $12,0(4,0)$ & $13,5(4,0)$ & $5,5(9,2)$ \\
\hline \multirow{8}{*}{$\begin{array}{l}\text { 6. Situações } \\
\text { de perda } \\
\text { urinária }\end{array}$} & Nunca & 0 & 1 & 0 & 2 \\
\hline & $\begin{array}{l}\text { Antes de chegar ao } \\
\text { banheiro }\end{array}$ & 5 & 2 & 3 & 1 \\
\hline & Quando tosse ou espirra & 4 & 3 & 4 & 2 \\
\hline & Quando está dormindo & 2 & 0 & 2 & 0 \\
\hline & Durante atividade física & 4 & 0 & 4 & 0 \\
\hline & Ao vestir-se após urinar & 0 & 0 & 1 & 0 \\
\hline & Sem razão óbvia & 2 & 0 & 2 & 0 \\
\hline & O tempo todo & 0 & 0 & 0 & 0 \\
\hline
\end{tabular}

Fonte: o autor.

*moda 


\section{Discussã̃o}

Nossos resultados demonstram que 10 sessões de cinesioterapia podem reduzir a frequência urinária das pacientes incontinentes, assim como favorecer o controle urinário, principalmente durante a realização de atividade física. Sugerem também que, quando associado à cinesioterapia, o aconselhamento (com informação e estímulo à continuidade do tratamento em casa, por meio de exercícios e diário miccional) pode reduzir a quantidade de urina perdida, a interferência da IU na vida diária e o impacto sobre a qualidade de vida de mulheres incontinentes.

A melhora identificada no GC pode ser justificada pelos efeitos derivados do fortalecimento muscular. Os músculos do assoalho pélvico são classificados histologicamente como músculos estriados e, como qualquer outro músculo esquelético, podem ser treinados para desenvolver aumento de força muscular e hipertrofia, o que acarreta em melhora da função e diminuição dos sintomas $^{20}$. O treinamento muscular é adquirido por meio da prática de exercícios específicos para o assoalho pélvico, baseados no preceito de que os movimentos voluntários repetidos proporcionam aumento da força muscular ${ }^{20}$. Seus efeitos benéficos incluem desenvolvimento, melhora, restauração ou manutenção, não só da força muscular como também da resistência, mobilidade, flexibilidade, relaxamento, coordenação e habilidade motora ${ }^{21}$.

Quanto ao aconselhamento sobre a IU, sabemos que este é preconizado ${ }^{22}$ e nosso estudo corrobora com essa prática, considerando que demonstrou melhores resultados no grupo que associou educação em saúde à cinesioterapia. Essas combinações de experiências de aprendizagem delineadas, com vistas a facilitar ações voluntárias conducentes à saúde ${ }^{18}$, apresentaram-se com grande valia nos nossos resultados.

Evidências anteriores confirmam a associação entre IU e baixa qualidade de vida ${ }^{23,24}$. Algumas características, como a presença de comorbidades, por exemplo, podem ter relação com a piora da qualidade de vida nas mulheres incontinentes e necessitam de maior investigação, sendo, então, esse assunto, sempre atual ${ }^{23}$. Felizmente, muitas estratégias de tratamento, farmacológicos e não farmacológicos, melhoram a IU e, consequentemente, a qualidade de vida, como observado em nosso estudo, especialmente no grupo que recebeu o aconselhamento ${ }^{23,24}$.

De maneira geral, aconselhamentos estimulam os indivíduos a adotarem e manterem padrões de vida sadios, a usarem de forma judiciosa os serviços de saúde à sua disposição e a tomarem suas próprias decisões, visando melhorar as suas condições de saúde e a do meio ambiente ${ }^{22}$. É possível que, considerando este estudo, as participantes do GCA tenham sido incentivadas pela conscientização sobre a IU, resultando em maior empenho ao tratamento.

Infelizmente, o aconselhamento não é realizado em grande parte dos tratamentos de IU e as dificuldades para desenvolver as ações educativas podem estar relacionadas ao comprometimento da equipe, adesão da comunidade, falta de recursos humanos, materiais e financeiros e falta de apoio por parte dos gestores ${ }^{25}$. É imprescindível que haja constante aprimoramento do conhecimento e prática dos profissionais para que 
as ações de educação em saúde possam ser desenvolvidas ${ }^{25}$.

Vale ressaltar que o aconselhamento, apesar de poder ser ofertado tanto individual como coletivamente, foi realizado em grupo no nosso estudo, e isso pode ter favorecido também os resultados. Uma revisão de 32 estudos experimentais controlados, os quais compararam as terapias de grupo e individual, concluiu que a terapia de grupo foi mais efetiva do que a individual em 25\% deles, e nos outros $75 \%$ não houve diferenças significativas ${ }^{18}$.

O fato do posicionamento de um cidadão se fazer em função do coletivo faz com que se tenha certeza de que os trabalhos em grupo e com ações interdisciplinares são essenciais na abordagem às adversidades exigidas pelo cuidado em saúde 3 . Portanto, o grupo tende a se tornar o espaço reflexivo que demanda a aprendizagem da convivência e o desenvolvimento de competências interpessoais, com compartilhamento de experiências. É fundamental o respeito ao senso comum dos membros, pois os aspectos cognitivos são indissociáveis dos afetivos. Técnicas de diálogo e problematização dos assuntos são aconselháveis, com indução de participação. Problematizar é responder as perguntas não com respostas, mas com novas indagações ${ }^{3}$.

A Fisioterapia é uma profissão da área da saúde capaz de desenvolver ações educativas para a promoção de saúde que interferem nos aspectos físicos, emocionais e sociais que influenciam na qualidade de vida dos indivíduos ${ }^{15}$. Com o objetivo de avaliar a eficácia do atendimento fisioterapêutico em grupo realizado em unidades básicas de saúde na melhora da qualidade de vida de seus usuários, um estudo avaliou 20 usuários participantes de atividades físicas e educativas em grupos coordenados por fisioterapeutas em quatro unidades básicas de saúde. Os resultados desse estudo demonstraram que as atividades físicas e educativas coordenadas pela fisioterapia promoveram uma melhora na capacidade funcional e no estado geral de saúde do indivíduo que são domínios relacionados à qualidade de vida ${ }^{26}$.

Não há dúvidas de que a maneira mais adequada de orientar o paciente seria por um profissional treinado em reabilitação pélvica, sendo que essas atividades preventivas e de educação sempre que possível, deve-se atuar nos fatores predisponentes e desencadeantes da IU ${ }^{26}$.

Acreditamos que resultados ainda melhores poderiam ser observados em uma amostra maior e com tempo prolongado, quando a educação em saúde consegue expressar suas progressivas influências.

\section{Conclusão}

É possível concluir que o aconselhamento uroginecológico nos casos de IU pode potencializar os benefícios derivados da cinesioterapia, auxiliando na diminuição da frequência e quantidade de urina perdida, reduzindo a interferência da IU na vida diária e melhorando a qualidade de vida das mulheres acometidas. 


\section{REFERÊNCIAS}

1. Lucas MG, Bosch RJ, Burkhard FC, Madden TB, Nambiar AK, Neisius A, et al. EAU guidelines on surgical treatment of urinary incontinence. European Urology. 2012; 62(6):1118-29

2. Pal M, Halder A, Bandyopadhyay S. Approach to a woman with urinary incontinence. Urol Ann. $2020 ; 12(1): 4-8$

3. Gomes ENM, Sousa AR, Rego A, Santos BC, Figueiredo CS, Gomes ENM, et al. Perfil de mulheres hipossuficientes com incontinência urinária participante e grupo de educação e promoção em saúde. Caderno de Educação, Saúde e Fisioterapia. 2016; 3(6)

4. Carneiro JA, Ramos GCF, Barbosa ATF, Medeiros SM, Lima CA, Costa FM, et al. Prevalência e fatores associados à incontinência urinária em idosos não institucionalizados. Cad. Saúde Colet. 2017; 25(3):268-77

5. Eshkoor SA, Hamid TA, Shahar S, Mun CY. Factors Related to Urinary Incontinence among the Malaysian Elderly. J Nutr Health Aging. 2017; 21(2):220-6

6. Mota R.L. Female urinary incontinence and sexuality. Int Braz J Urol. 2017; 43(1):20-8.

7. Gascón MRP, Mellão MA, Mello SH, Negrão RM, Casseb J, Oliveira ACP. The impact of urinary incontinence on the quality of life and on the sexuality of patients with HAM/TSP. Braz J Infect Dis. 2018; 22(4):288-93

8. Stickley A, Santini ZI, Koyanagi A. Urinary incontinence, mental health and loneliness among community-dwelling older adults in Ireland. BMC Urol. 2017; 17(1):29.

9. Lim YM, Lee SR, Choi EJ, Jeong K, Chung HW. Urinary incontinence is strongly associated with depression in middle-aged and older Korean women: Data from the Korean longitudinal study of ageing. Eur J Obstet Gynecol Reprod Biol. 2018; 220:69-73

10. Fonseca ESM, Camargo ALM, Castro RA, Sartori MGFS, Fonseca MCM, Lima GR, et al. Validação do questionário de qualidade de vida (King's Health Questionnaire) em mulheres brasileiras com incontinência urinária. Rev. Bras Ginecol Obstet. 2005; 27(5):235-42

11. Basak T, Kok G, Guvenc G. Prevalence, risk factors and quality of life in Turkish women with urinary incontinence: a synthesis of the literature. Int Nurs Rev. 2013; 60(4):448-60

12. Mostafaei H, Sadeghi-Bazargani H, Hajebrahimi S, Salehi-Pourmehr H, Ghojazadeh M, Onur $\mathrm{R}$, et al. Prevalence of female urinary incontinence in the developing world: A systematic review and meta-analysis-A Report from the Developing World Committee of the International Continence Society and Iranian Research Center for Evidence Based Medicine. Neurourol Urodyn. 2020; 39(4):1063-86

13. Henkes DF, Fiori A, Carvalho JAM, Tavares KO, Frare JC. Incontinência urinária: o impacto na vida de mulheres acometidas e o significado do tratamento fisioterapêutico. Semina cienc. biol. saúde. 2015; 36(2):45-56

14. Berquó MS, Ribeiro MO, Amaral RG. Fisioterapia no tratamento da incontinência urinária feminina. Rev Femina. 2009; 37(7):385-88

15. Kisner C, Colby LA, Borstad J. Exercícios Terapêuticos: fundamentos e técnicas. 7a ed. São Paulo: Manole; 2021.

16. Baracho E. Fisioterapia aplicada à saúde da mulher. 6a ed. Rio de Janeiro: Guanabara Koogan; 2018.

17. Moreno AL. Fisioterapia em uroginecologia. 2a ed. Barueri: Manole; 2009.

18. Gusso G, Lopes JMC, Dias LC. Tratado de Medicina de Família e Comunidade: princípios, formação e prática. 2. ed. Porto Alegre: Artmed Editora Ltda; 2018.

19. Irwin GM. Urinary Incontinence. Prim Care. 2019; 46(2):233-42 
20. Huang YC, Chang KV. Kegel Exercises. 2021 May 9. In: StatPearls [Internet]. Treasure Island (FL): StatPearls Publishing; 2021

21. Dumoulin C, Cacciari LP, Hay-Smith EJC. Pelvic floor muscle training versus no treatment, or inactive control treatments, for urinary incontinence in women. Cochrane Database Syst Rev. 2018; 10(10):CD005654

22. Huang Z, Wu S, Yu T, Hu A. Efficacy of telemedicine for urinary incontinence in women: a systematic review and meta-analysis of randomized controlled trials. Int Urogynecol J. 2020; 31(8):1507-13

23. Pizzol D, Demurtas J, Celotto S, Maggi S, Smith L, Angiolelli G, et al. Urinary incontinence and quality of life: a systematic review and meta-analysis. Aging Clin Exp Res. 2021; 33(1):25-35

24. Vaughan CP, Markland AD. Urinary Incontinence in Women. Ann Intern Med. 2020; 172(3):ITC17-ITC32

25. Andrade ACV, Schwalm MT, Ceretta LB, Dagostin VS, Soratto MT. Planejamento das ações educativas pela equipe multiprofissional da Estratégia Saúde da Família. O Mundo da Saúde. 2013; 37(4):439-49

26. Aquino CF, Augusto VG, Moreira DS, Ribeiro S. Avaliação da qualidade de vida de indivíduos que utilizam o serviço de fisioterapia em unidades básicas de saúde. Fisioter Mov. 2009; 22(2):271-9

\section{DADOS DOS AUTORES}

\section{Carolina Nascimben Matheus}

Doutora em Ciências Médicas; Faculdades Integradas Einstein de Limeira,

Limeira/SP - Brasil; ca_nm@yahoo.com.br

\section{Cintia Furlan}

Doutora em Ciências Médicas; Universidade Estadual de Campinas (UNICAMP), Campinas/SP - Brasil; cin_furlan@yahoo.com.br

\section{Paloma Felício de Oliveira Lima}

Especialista em fisioterapia pélvica uroginecológica; Faculdades Integradas Einstein de Limeira, Limeira/SP - Brasil; fisio.pfelicio@yahoo.com.br

\section{Milene Teles Pereira}

Fisioterapeuta; Faculdades Integradas Einstein de Limeira, Limeira/SP - Brasil; milenetelisp@gmail.com

\section{Paloma Bernardelli dos Santos}

Fisioterapeuta; Faculdades Integradas Einstein de Limeira, Limeira/SP - Brasil; bernardellipaloma@gmail.com

Submetido em: 1-11-2019

Aceito em: 13-4-2021 